\title{
Ten-year outcomes of antivascular endothelial growth factor therapy in neovascular age-related macular degeneration
}

\author{
Shruti Chandra $\mathbb{D}^{1} \cdot$ Cristina Arpa $^{1} \cdot$ Deepthy Menon ${ }^{1} \cdot$ Hagar Khalid $\mathbb{D}^{1} \cdot$ Robin Hamilton $^{1} \cdot$ Luke Nicholson $^{1}$. \\ Bishwanath Pal ${ }^{1} \cdot$ Sandro Fasolo $^{1} \cdot$ Philip Hykin $^{1} \cdot$ Pearse A. Keane ${ }^{1} \cdot$ Sobha Sivaprasad $^{1}$
}

Received: 6 October 2019 / Revised: 6 January 2020 / Accepted: 9 January 2020 / Published online: 24 January 2020

(c) The Author(s), under exclusive licence to The Royal College of Ophthalmologists 2020

\begin{abstract}
Purpose Single center, noninterventional cohort study to assess 10-year visual and anatomical outcomes following initiation of treatment with antivascular endothelial growth factor (anti-VEGF) agents in neovascular age-related macular degeneration (AMD) patients. Neovascular AMD patients initiated on intravitreal anti-VEGF injections in 2008-2009 and continued to be followed up for at least 10 years were included in this study.

Methods The Moorfields OpenEyes database was searched for all patients who were initiated on anti-VEGF therapy for neovascular AMD in 2008-2009 and the visual acuity (VA) in Early Diabetic Retinopathy Study (ETDRS) letters and injection records were analyzed for those who have had at least 10-year follow-up. The spectral-domain optical coherence tomography (SD-OCT) scans, color fundus photos, and fundus fluorescein angiography (FA) were graded by two retinal physicians. The outcomes were also compared between those with good and poor VA outcomes based on pre-defined criteria. The primary end point was change in VA at 10 years; secondary outcomes included percentage with VA of 20/40 or better, 20/70 or better, VA gains and losses, anatomic outcomes and number of injections.

Results After a mean of 10.04 years after initiation of anti-VEGF therapy, the mean decline in VA from baseline was -2.1 ETDRS letters (SD 19.9, $p=0.65$ ). One hundred eyes (67.1\%) achieved a VA threshold of 20/70 or better, 33.5\% achieved a VA of 20/40 or better, and $76.5 \%$ eyes maintained VA defined as a loss of less than 15 letters. Fourteen percent of study eyes had VA of 20/200 or worse and $23.5 \%$ declined by 15 letters or more. $87.5 \%$ of eyes were switched from ranibizumab to aflibercept during the course of 10 years and the eyes received a mean of 52.2 (SD 18.1) injections over 10 years. From this cohort, 87 (58.3\%) eyes are having on-going treatment. On OCT, 34.9\% had persistent fluid at the last visit, 6.7\% patients showed new onset atrophy compared to baseline, and $43.7 \%$ had increased area of macular atrophy. The mean area of atrophy at the final visit was $4.15 \mathrm{~mm}^{2}$. Comparison between the good and worse visual outcome groups showed lower baseline VA, fovea-involving atrophy and final area of atrophy had a statistically significant negative effect on the final visual outcome $(p<0.05)$.

Conclusions Regular monitoring and anti-VEGF treatment over 10 years reduce the risk of visual loss of 15 letters or more in patients with neovascular AMD. The most common cause of substantial visual decline was macular atrophy.
\end{abstract}

\section{Introduction}

Neovascular age-related macular degeneration (nAMD) remains a common cause of visual impairment in people aged 55 years or older. Without treatment, this condition

Shruti Chandra

Shruti.chandra@nhs.net

1 National Institute of Health Research Moorfields Biomedical Research Centre, Moorfields Eye Hospital, London, UK progresses and results in irreversible central visual loss of an average of ten letter loss within the first 12 months [1]. In the last decade, the introduction of intravitreal antivascular endothelial growth factor (anti-VEGF) agents has revolutionized the treatment of wet AMD, offering patients with previously unachievable improvement in vision. Bevacizumab (Avastin; Genentech, Inc.) was the first panVEGF-A inhibitor to be used as an off-label treatment option for nAMD [2]. Although bevacizumab is used widely around the world, this agent is not recommended for use in the National Health Service (NHS) in the United Kingdom (UK) due to the unlicensed and off-label use of 
the drug. In 2005, clinical trials established the efficacy of ranibizumab (Lucentis; Genentech, Inc.) for the treatment of this condition followed by aflibercept (Eylea; Regeneron, Inc.) in 2012 [1, 3, 4]. These agents have since been the treatment of choice for nAMD in the UK.

Intravitreal anti-VEGF agents decrease vascular leakage and are antiangiogenic. However, disease activity may remain for years due to their short duration of action and inability to cause involution of mature vessels. It remains unclear whether these agents may in the long-term also cause macular atrophy (MA). The comparison of AMD treatment trials (CATT) research group and Seven-Year Observational Update of Macular Degeneration Patients Post-MARINA/ANCHOR and HORIZON Trials (SEVENUP) study have tried to tease out the associations of visual decline by analyzing long-term visual and anatomical outcomes of eyes with nAMD recruited into anti-VEGF treatment trials $[5,6]$.

In the CATT study, almost $50 \%$ of the patients had a visual acuity (VA) of 20/40 or better, however the visual gains at the end of first 2 years were not maintained at 5 years. Similarly, in the SEVEN-UP study, half of the eyes continued to remain stable but almost one-third declined by 15 letters or more [5, 6]. Thus, these studies not only confirm the long-term therapeutic advantage offered by anti-VEGF therapy for nAMD but also show that eyes with exudative AMD continue to remain at risk even in the late stages of therapeutic course.

All across the world, variations in therapy application, differences in anti-VEGF agent used, and inconsistencies in treatment guidelines have led to varying outcomes, with real-life outcomes $[7,8]$ being inferior to those from clinical trials. The NHS treats patients with nAMD according the recommendations of the National Institute for Health and Care Excellence and guidelines produced by the Royal College of Ophthalmologists. The aim of the current study was to assess the long-term outcomes within a cohort of neovascular AMD patients on follow-up for 10 years post initiation of anti-VEGF therapy.

\section{Methods}

\section{Study design}

Data on this retrospective cohort were extracted from the Electronic Medical records database of Moorfields Eye Hospital, London, UK. We included consecutive patients with nAMD initiated on anti-VEGF therapy in 2008-2009 who had at least 10 years of follow-up. Data collected included patient demographics, clinic-based VA at baseline and follow-up at 10 years (Snellen VA, if used, was converted to Early Treatment Diabetic Retinopathy Study
(ETDRS) letters for purposes of statistical analysis), antiVEGF agents used, and number of injections. Findings on color fundus photographs (CFP) and spectral-domain optical coherence tomography (SD-OCT) were re-graded at baseline and 10-year follow-up while fluorescein angiograms (FA) were assessed at baseline only. Complete data were defined as documented VA, number and type of injections and gradable OCT scans (signal strength $>7$ ) at baseline and 10-year follow-up. The date of first injection was defined as baseline date and the last follow-up visit to the clinic was considered as final follow-up.

The study adhered to the tenets of Declaration of Helsinki and was approved by the Clinical Effectiveness Committee at the hospital (CA18/MR/15-141).

\section{Study cohort}

A total of 149 eyes of 149 patients were included in the study. The process of study enrollment and allocation has been shown in the CONSORT diagram (Fig. 1).

The eyes were further divided into two groups based on the letter change in VA from baseline to final visit. Group A (good visual outcomes) - eyes with gain of 10 letters or more; Group B (worse visual outcomes)—eyes with loss of 15 letter or worse.

\section{Treatment protocol}

The treatment protocol from 2008 to 2013 recommended three loading doses of ranibizumab followed by pro-re-nata (PRN) regimen. This was changed in 2013 after introduction of aflibercept in the NHS for treatment of neovascular AMD. This treatment protocol is currently followed as well and recommended three loading doses followed by 8 weekly fixed dosing until week 40 when the patients could be transferred to a treat and extend regimen. The treat and extend regimen allowed visits to be extended at 2-4 weekly intervals and reduced by 2 weeks in case of reactivation. When patients were injected at 12 weekly intervals for three consecutive visits without any evidence of reactivation, they could be referred to a stable AMD retinal clinic where they would be monitored 8-12 weekly with VA and OCT measurements. If they show any signs of activation, the patients are reinitiated on a treat and extend regimen. Some patients may receive a PRN dosing according to clinician discretion

\section{Outcome measures}

The primary outcome measure was change in VA over 10 years of treatment. Secondary outcome measures included, the percentage of patients with Snellen equivalent BCVA of $20 / 40$ or better in the study eye and patients with $20 / 70$ or 
Fig. 1 Consort diagramcohort study. retrospective nonrandomized

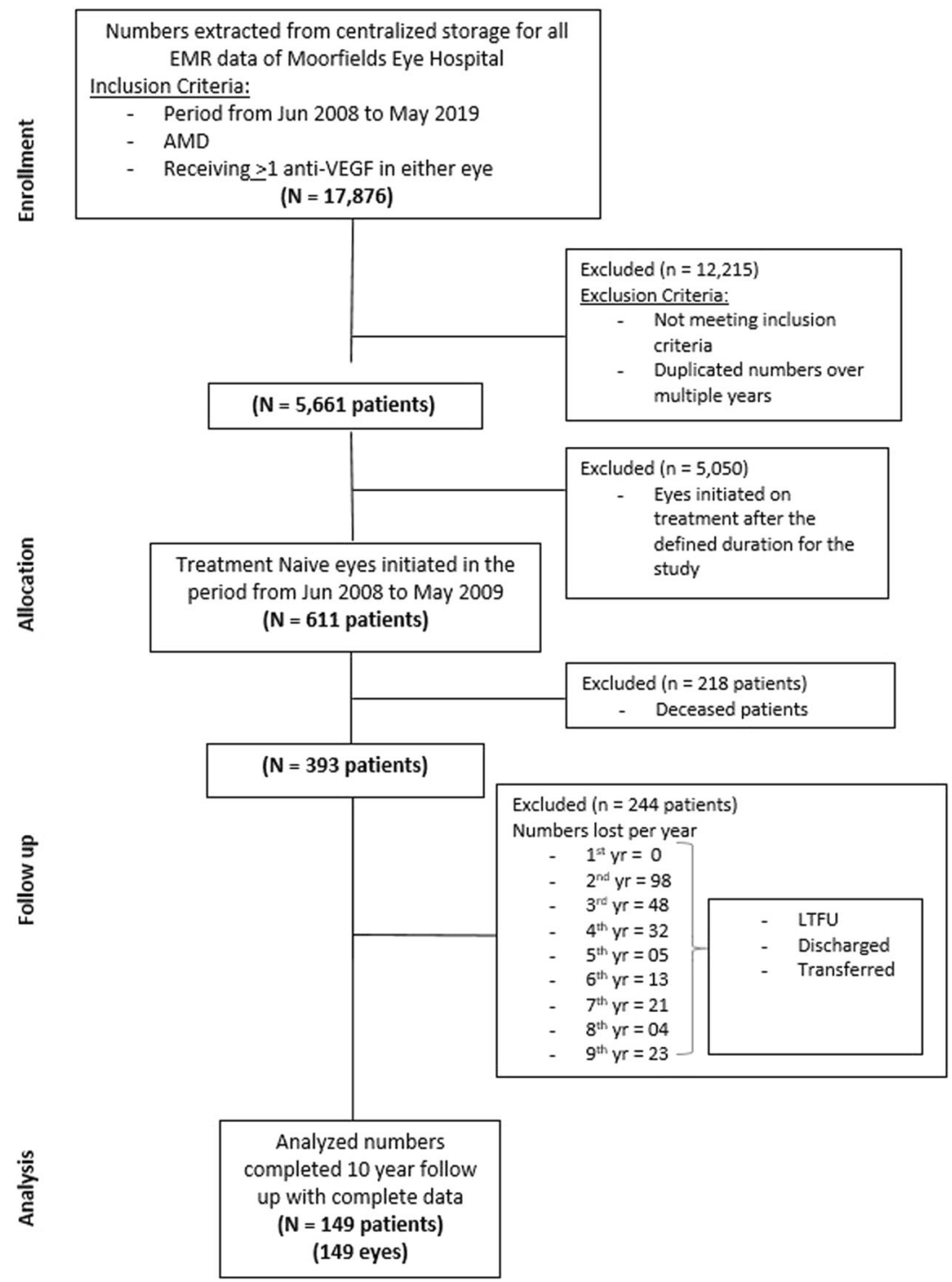

\section{Definition of total retinal thickness at fovea} ETDRS vision testing, percentage of eyes with disease quiescence (defined as dry scan on OCT), change in total retinal thickness at fovea, percentage of eyes with foveal atrophy, area of central atrophy at final visit, comparative analysis between Group A and Group B. Change in ETDRS letter score was calculated between the baseline and final visit for each patient individually.

\section{Image analysis}

Images (SD-OCT, CFP, and FA) were graded by two retinal physicians (SC and DM). Main anatomic outcomes for the purpose of this study included the presence of fluid (intraretinal or subretinal), total retinal thickness at fovea on SD-OCT, presence and size of MA on CFP both at baseline and final visit. The presence and type of leakage on FA was documented at baseline only.
The total retinal thickness included the sum of retinal thickness, subretinal fluid, and subretinal tissue in the central subfield. It was measured in microns at the foveal center.

\section{Determination of macular atrophy}

MA was defined on CFP (50 degrees Topcon color fundus photo) as one or more discrete areas of the loss of retinal pigment epithelium, within the macular vascular arcades of $\geq 250 \mu \mathrm{m}$ with a color and thickness change relative to the surrounding retina, and more prominent visualization of the choroidal vessels [9]. Foveal MA was defined as area of MA involving a circle of $500 \mu \mathrm{m}$ radius centered at the fovea. Nonfoveal MA was defined as areas of atrophy that did not meet the definition of foveal MA. Trained graders outlined the perimeter of all qualifying MA lesions using the freehand 
drawing tool in ImageJ (NIH, Bethesda, MD). If multiple MA lesions existed, they were traced separately and summed.

\section{Statistical analysis}

Data were collected from the electronic medical records and entered manually onto an excel sheet. Data were analyzed using SPSS Statistics for Windows, Version 24.0. Armonk, NY: IBM Corp. Shapiro-Wilk test was used to check for normality of the data and the data were found to be not normally distributed. Wilcoxon signed rank test was used to compare means at baseline and final follow-up. Mann-Whitney $U$-test was used for comparisons between Group A and Group B. Chi-square test was used to explore differences in proportions among categorical data in independent groups. A $p$ value of $<0.05$ was considered statistically significant.

\section{Results}

\section{Study population and baseline characteristics}

The study cohort consisted of 149 eyes of 149 patients. The mean age in years was $74.5( \pm 7.8)$ and almost two-third of the cohort was female. Seventy-five percent of patients were Caucasians. The mean duration of follow-up was 3667.4 $( \pm 15)$ days or 10.04 (range, 10-10.08) years.

Baseline FA were available for 147 eyes. Nearly all FAs $(91.3 \%)$ showed presence of leakage with the predominant lesion type being occult $(40.4 \%)$, followed by minimally classic (38.2\%) and least common was a classic type leak $(19.1 \%)$. Retinal angiomatous proliferation was diagnosed in three eyes and there were no eyes with polypoidal choroidal vasculopathy in this cohort. There were seven eyes where due to poor image quality it was difficult to assess the presence and type of leakage. These eyes were graded as questionable.

Mean VA at baseline was $59.5( \pm 13.1)$ ETDRS letters. Baseline mean foveal thickness was $298.7( \pm 87.9) \mu \mathrm{m}$. All the eyes showed presence of fluid (intraretinal or subretinal or both) at baseline. Fifteen eyes had atrophy at baseline (11 nonfoveal and 4 foveal) and the mean area of atrophy was $0.11 \mathrm{~mm}^{2}$.

\section{Vision outcomes}

As shown in Table 1, mean change in VA from baseline in ETDRS letters in the whole cohort was -2.1 letters (SD $19.9, p=0.65)$. One hundred eyes $(67.1 \%)$ of 149 study eyes achieved a VA threshold of $20 / 70$ or better. Good vision defined as final BCVA of 20/40 or better was observed in $50(33.5 \%)$ eyes. Twenty eyes (14\%) were
Table 1 Vision outcomes for all eyes at baseline and at 10-year follow-up.

\begin{tabular}{|c|c|c|c|}
\hline Outcome & Baseline & $\begin{array}{l}\text { At } 10 \text {-year } \\
\text { follow-up }\end{array}$ & $p$ value \\
\hline \multicolumn{4}{|c|}{ Visual acuity score, letters Snellen equivalent, no. (\%) } \\
\hline & $N=149$ & $N=149$ & \\
\hline $83-97,20 / 12-20 / 20$ & $3(2)$ & $3(2)$ & 1 \\
\hline $68-82,20 / 25-20 / 40$ & $55(36.9)$ & $47(31.5)$ & 0.39 \\
\hline $53-67,20 / 50-20 / 80$ & $56(37.5)$ & 50 (33.6) & 0.54 \\
\hline $38-52,20 / 100-20 / 160$ & $20(13.4)$ & $28(18.8)$ & 0.27 \\
\hline $37-18,20 / 200-20 / 400$ & $15(10)$ & $18(12)$ & 0.71 \\
\hline$<17,<20 / 400$ & $0(0)$ & $2(2)$ & 0.16 \\
\hline Mean letters (SD) & $59.5(13.1)$ & $57.4(17.8)$ & 0.57 \\
\hline $\begin{array}{l}\text { Mean change in visual } \\
\text { acuity (SD) }\end{array}$ & $-2.1(19.9)$ & & \\
\hline \multicolumn{4}{|c|}{ Change in visual acuity score, from baseline, letters, no. (\%) } \\
\hline \multicolumn{4}{|l|}{15 letter change } \\
\hline$>15$ increase & $29(19.5)$ & & \\
\hline $5-14$ increase & $34(22.8)$ & & \\
\hline$<4$ change & $26(17.5)$ & & \\
\hline 5-14 decrease & $25(16.7)$ & & \\
\hline 15-29 decrease & $19(12.8)$ & & \\
\hline$>30$ decrease & $16(10.7)$ & & \\
\hline
\end{tabular}

legally blind in the study eye, defined as final BCVA of 20/ 200 or worse. One hundred and fourteen eyes (76.5\%) maintained VA defined as less than 15 letters loss from baseline.

A gain of more than 10 letters was noted in 37 (24.8\%) eyes as against 41 eyes (27.5\%) that lost more than 10 letters. Decline of 15 letters or more was seen in $23.5 \%$ eyes of which, 16 (10.7\%) eyes showed marked drop in VA of 30 or more letter decrease. The change in VA in ETDRS letters was calculated individually for each patient and the data are shown in the form of a waterfall chart (Fig. 2). The values ranged from a maximum gain of 45 letters to a maximum loss of 66 letters.

\section{Injection outcomes}

The total number of anti-VEGF injections received from the initiation of therapy was calculated individually for each eye. The mean number of injections received over the entire period of follow-up was $52.2( \pm 18.1)$, ranging from 15 to 98 injections for the entire cohort. One hundred and thirty-one eyes $(87.9 \%)$ received ranibizumab injection followed by switch to aflibercept. Three eyes received all the three drugs (bevacizumab, ranibizumab, and aflibercept). Only 11 eyes (7.4\%) that were injected with ranibizumab monotherapy over 10 years. The remaining four eyes received various combinations of the three anti-VEGF agents. Eyes receiving more than the mean of 52 injections had a mean final VA of $58.0( \pm 19.5)$ letters and those receiving less than 52 injections had a final VA of $56.9( \pm 16.2)$. The difference between the two was not statistically significant $(p=0.70)$. 
Fig. 2 Chart showing the letter change in VA per patient from baseline to final visit.

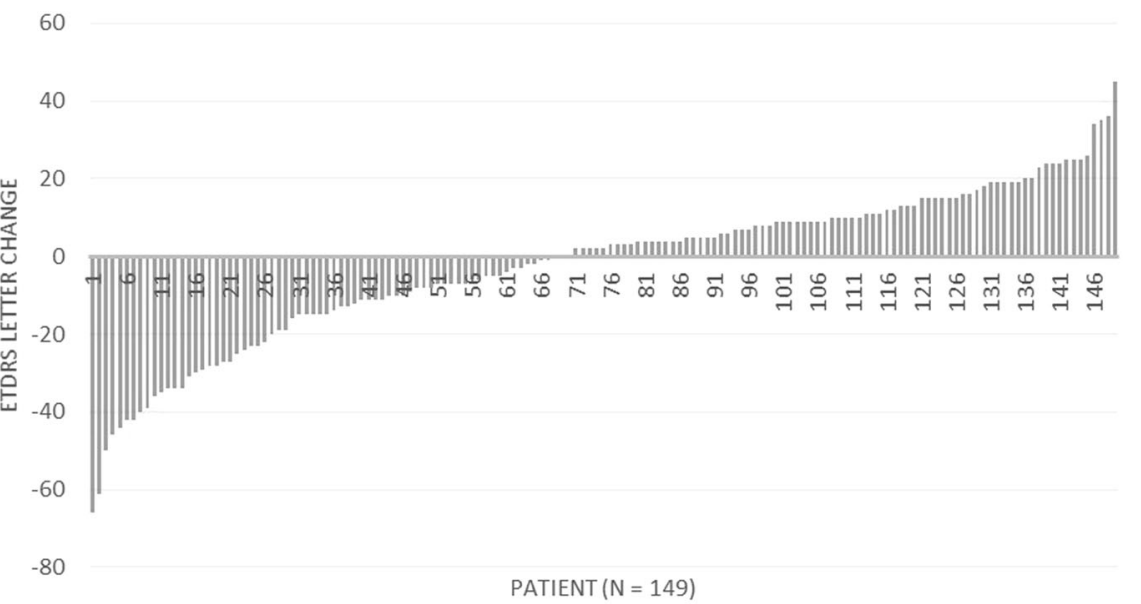

\section{Anatomic outcomes}

SD-OCT scans were available for all patients at baseline and final follow-up. The OCT scans were evaluated for total retinal thickness at fovea (in microns) and presence of fluid (Table 1). The mean foveal thickness at final follow-up was $206.9( \pm 54.9) \mu \mathrm{m}$, a decrease of $91.7( \pm 106.3) \mu \mathrm{m}$ from baseline ( $p=0.0001)$. While comparing the presence of any fluid (intraretinal/subretinal) on SD-OCT, all 149 eyes $(100 \%)$ had fluid at baseline which reduced to 52 eyes $(34.9 \%)$ at final visit $(p=0.0001)$. Twenty-four eyes had intraretinal fluid only, 20 eyes had subretinal fluid alone and 8 eyes had both. Color fundus photographs showed increase in number of eyes with foveal atrophy from $4(2.7 \%)$ at baseline to $22(14.8 \%)$ at final visit $(p=0.0001)$. Of these 22 eyes that had foveal atrophy $t 10$ years, 4 eyes had subfoveal atrophy at baseline, and 8 eyes had nonfoveal atrophy at baseline that expanded to involve the fovea by final visit. New onset subfoveal atrophy was seen in 10 (6.7\%) eyes. Additionally, the number of eyes with nonfoveal atrophy increased from $11(7.4 \%)$ at baseline to 43 (28.9\%) eyes at final follow-up. The proportion of eyes with any form of atrophy at final visit was $43.6 \%$. The total area of subfoveal atrophic lesion at final follow-up was $4.15 \mathrm{~mm}^{2}$, an increase from $0.11 \mathrm{~mm}^{2}$ at baseline. This increase was statistically significant $(p=0.0001)$. Subretinal fibrosis was seen in 9 eyes at baseline that increased to $44(29.5 \%)$ eyes at final follow-up.

\section{Comparative analysis between Group A and Group B}

The cohort was divided on the basis of change in VA (ETDRS letters) into group with good outcomes (gain of 10 letters or better), Group A $(n=37)$ and worse outcomes (loss of 15 letters or worse), Group B $(n=35)$. The visual and anatomic outcomes of the two groups are shown in Table 2. The difference in means of baseline VA between the groups was statistically significant with patient in Group A having better baseline vision than those in the Group B $(p=0.0001)$. The mean age and mean number of injections were almost similar in both groups ( $p$ value 0.56 and 0.28 , respectively). Among the morphological outcomes the final mean foveal thickness and change in foveal thickness from baseline between the two groups was not statistically significant ( $p$ value 0.16 and 0.96 , respectively). The presence of fluid (both IRF and SRF) was not significantly different between the two groups $(p=0.12)$. However, there were significantly more eyes with foveal atrophy in the worse outcome group $(p=0.0001)$. In accordance with this, there were more eyes without atrophy (23) in Group A than (10) Group B $(p=0.01)$. At baseline there was no statistically significant difference in mean atrophy area between the two groups. However, the mean area of final atrophy was more in Group B at $8.5( \pm 5.5)$ $\mathrm{mm}^{2}$ versus $2.5 \quad( \pm 4.2) \mathrm{mm}^{2} \quad(p=0.004)$. Thus, worse visual outcomes significantly correlated with better baseline VA, presence of foveal atrophy at final visit and increased area of atrophy at final visit.

\section{Cohort with incomplete data}

The mean VA of eyes with incomplete follow-up per year was also analyzed. The mean VA at baseline and final visit per year is shown in Table 3. The mean change in VA in this cohort per year varied with maximal VA loss observed in the group that discontinued in year 3-6, VA gains noted in 1-2 years, and stable VA from year 7 onwards.

\section{Safety outcomes}

This study did not look at safety concerns as an outcome. However the 1-year endophthalmitis rate as documented by internal audits was 1:4000 for intravitreal injections in our hospital. 
Table 2 Morphological outcomes for Group A (gain of 10 or more letters) versus Group B (loss of 15 or more letters).

\begin{tabular}{|c|c|c|c|}
\hline Outcome & Group A $(n=37)$ & Group B $(n=35)$ & $p$ value \\
\hline \multicolumn{4}{|c|}{ Baseline visual acuity ETDRS letters } \\
\hline Mean (SD) & $71.4(10.5)$ & $66.6(8.84)$ & 0.00001 \\
\hline \multicolumn{4}{|l|}{ Age (years) } \\
\hline Mean (SD) & $73.7(8.2)$ & $74.7(8.1)$ & 0.56 \\
\hline \multicolumn{4}{|c|}{ Mean no. of injections } \\
\hline Mean (SD) & $48.2(17.8)$ & $53.4(19.2)$ & 0.28 \\
\hline \multicolumn{4}{|c|}{ Final total thickness at fovea, $\mathrm{mm}$} \\
\hline Mean (SD) & $220.5(56.5)$ & $197.4(56.1)$ & 0.16 \\
\hline Mean change (SD) & $-82.0(90.6)$ & $-88.7(85.1)$ & 0.96 \\
\hline \multicolumn{4}{|c|}{ Final fluid on optical coherence tomography; no. (\%) } \\
\hline Absent & $20(54.0)$ & $26(74.3)$ & 0.12 \\
\hline Present & $17(45.9)$ & $9(25.7)$ & \\
\hline IRF & $10(27.0)$ & $5(14.3)$ & 0.87 \\
\hline SRF & 7 (18.9) & $4(11.4)$ & \\
\hline \multicolumn{4}{|c|}{ Final geographic atrophy; no. (\%) } \\
\hline Absent & $23(62.2)$ & $10(28.6)$ & 0.01 \\
\hline \multicolumn{4}{|l|}{ Present } \\
\hline Nonfoveal & $13(35.1)$ & $6(17.1)$ & \\
\hline Foveal & $0(0)$ & $17(48.6)$ & 0.0001 \\
\hline Unknown/missing & $1(2.7)$ & $2(5.7)$ & \\
\hline \multicolumn{4}{|c|}{ Final area of lesion, $\mathrm{mm}^{2}$} \\
\hline Mean (SD) & $2.5(4.2)$ & $8.5(5.5)$ & 0.004 \\
\hline Mean change (SD) & $2.5(4.2)$ & $8.3(5.3)$ & 0.004 \\
\hline
\end{tabular}

\section{Discussion}

This study was conducted to determine the 10 -year realworld outcomes of patients initiated on anti-VEGF agents and is still adhering to the treatment regimens and monitoring in a hospital care setting, perhaps highlighting the cohort of patients that most benefited from this intensive and challenging therapy. As clinical trials do not extend for such long periods, this study provides an insight into the course of treated neovascular AMD in the real-world scenario at 10 years. The treatment regimens with anti-VEGF have changed over this long period. Although all patients are still initiated on a loading phase of three injections, the treatment regimens have changed from PRN ranibizumab therapy to a fixed dosing aflibercept therapy, which is then weaned off to a treat and extend protocol.

The primary outcome measure for this study was the change in VA from baseline in treatment naive patients. Based on the aforementioned interventions and regimens, the mean change in VA was -2.1 letters. The outcomes suggest that a significant portion of patients on follow-up remained stable over 10 years. Two-thirds $(67.1 \%)$ of the cohort had VA of 20/70 or better with one-third of patients (33.5\%) having a good vision of $20 / 40$ or better after a
Table 3 Visual outcomes for patients lost to follow-up per year over 10 years.

\begin{tabular}{lcll}
\hline $\begin{array}{l}\text { Year patient lost } \\
\text { to follow-up }\end{array}$ & $\begin{array}{l}\text { No. of } \\
\text { patients }\end{array}$ & VA at baseline & VA at final visit \\
\hline 1st & 0 & $55.5(10.2)$ & $73.1(12.3)$ \\
2nd & 98 & $61.0(11.8)$ & $85.3(7.9)$ \\
3rd & 48 & $70.2(17.1)$ & $65.9(14.5)$ \\
4th & 32 & $62.8(12.4)$ & $53.7(11.3)$ \\
5 th & 5 & $70.1(15.1)$ & $62.2(11.7)$ \\
6 th & 13 & $61.9(9.8)$ & $44.3(10.8)$ \\
7 th & 21 & $59.3(15.3)$ & $57.0(14.4)$ \\
8th & 4 & $58.7(10.6)$ & $55.8(16.9)$ \\
9th & 23 & $57.5(9.9)$ & $54.7(16.9)$ \\
\hline
\end{tabular}

decade of therapy. These results were in accordance with the SEVEN-UP study that reported a VA of $20 / 70$ or better in one-third of patients and a quarter of patients with VA 20/40 or better at the end of a 7.3 year follow-up [6]. However, these patients continue to remain at risk of visual decline due to persistent disease activity, even in the later stages of the disease thereby, necessitating the need for continued antiVEGF therapy. About $14 \%$ patients were legally blind in the study eye with a final VA of 20/200 or worse.

The overall decline in vision however, was higher $(8.2$ letters) in the SEVEN-UP study. This discrepancy could be explained by the differences in baseline characteristics of the cohort in our study versus those in the SEVEN-UP study. The mean baseline VA in letters was lower in the SEVEN-UP study (54.3 letters) than our study (59.5 letters). Moreover, the eyes in the worse VA groups of 20/200 or worse at baseline were as high as $36.9 \%$ in SEVEN-UP as against $10 \%$ in our cohort. Patient heterogeneity may also play a role as most of the patients in SEVEN-UP study were nonwhite vis-à-vis our cohort with $75 \%$ Caucasian population. The healthcare systems also prove to contribute to final visual outcomes [7]. Treatment is free at the point of care in the NHS.

On OCT imaging, almost two-thirds of eyes had a dry macula at final follow-up. The mean final retinal thickness was also significantly thinner than the baseline. These findings indicate that a dry retina is an achievable end point over an extended period of treatment and regular follow-up. However, increase in MA is almost inevitable with $6.7 \%$ eyes having new onset atrophy and $43.7 \%$ eyes having increase in atrophy at final visit. All these factors contribute to poor final visual outcome with a mean loss in VA letters even with protocol-directed management. Increased area of MA and presence of subfoveal MA has been previously shown to negatively impact the final VA [6].

Overall mean number of injections received was 52.2 over a period of 10 years. Previous studies on long-term 
outcomes at 1, 2, 5, and 7 years have all shown the reduced effectiveness of anti-VEGF agents in clinical practice and most reports have attributed these results to the reduced number of injections over time [5, 6, 10-13]. Qin et al. in their study on long-term outcomes of exudative AMD treatment have attributed the variable results to the differences in frequency and regularity of anti-VEGF treatment [14]. The extended CATT study and the VIEW 1 extension study concluded a direct association between the number of injections and final VA with more number of injections resulting in better VA outcomes [15].

In our cohort, the eyes that lost vision received more injections when compared to the eyes that gained VA letters. The eyes in worse VA group had statistically insignificant higher number of injections but also had significantly higher proportions of foveal atrophy and larger final area of atrophic lesion. These findings indicate that despite protocol driven treatment regimens over years, the natural history of the disease of MA does influence VA. The IVAN study concluded a definite relation between the development of geographic atrophy and the dosing regimen of anti-VEGF injections. At the end of 2 years, the risk of developing geographic atrophy was reported as $34 \%$ with monthly injections versus $26 \%$ with PRN administration [16]. In the CATT trial as well, the area of atrophy at end of 2 years was $21.1 \%$ versus $11.5 \%$ in the PRN group [17]. The HARBOR study group also concluded that presence of subretinal fluid decreased the risk for developing geographic atrophy so aggressive regimens to dry the retina should be avoided, indirectly indicating that fewer injections aid in avoiding final geographic atrophy [18]. The question of whether in the long-term higher anti-VEGF therapy contributes to development of or increase in area of MA remains unanswered [19]. However, this study shows that benefits of anti-VEGF therapy outweigh risks of MA in most patients in the cohort.

Another finding of interest while comparing the two groups was the baseline VA. Baseline VA was a statistically significant factor contributing to final visual outcome with gainers having higher baseline VA. Baseline VA thus according to our results is an additional determinant of final VA outcome. This result, however, is in contrast to the phenomenon of ceiling effect wherein higher baseline VA lessens the estimated gain in VA [13]. However, ceiling effect is only applicable to eyes that has limited potential for improvement. This study highlights that eyes with presenting VA below the mean of 52 letters have less potential for improvement and may already have permanent structural changes that are not identifiable with current imaging devices. Patient factor could also play a role with patients having better baseline VA being motivated to preserve VA thereby compliant to regular treatments and attending regular follow-up visits.

The other findings that were observed in the comparative analysis between the groups were the absence of effect of age at diagnosis on final VA. Among the morphological outcomes, the percentage of eyes with dry retina was higher in the worse outcome group. In the present scenario of OCT directed therapy management, it is interesting to see that a dry retina did not have a significant bearing on the final visual outcome. On the contrary, the results indicate otherwise with dry retina being associated with greater VA loss.

The strengths of this study include that it is the first report of a 10-year outcome in the UK NHS system where cost of drug does not influence outcome. The AURA study showed that anti-VEGF services run in the UK are one of the best services in the world with patients being monitored regularly with ETDRS vision testing and OCT. Also the treatment regimens are protocol driven [13]. Therefore, these study results, although inferior to clinical trial settings, are as robust as possible. To eliminate confounders, only treatment naive eyes were included in the study. The selection of patients was done in a systematic manner accounting for each patient, with recording of numbers lost to follow-up per year.

The limitations are that the study is retrospective and the study only included patients who were on follow-up for 10 years representing a best cared population. The retention rate was good with 244 patients lost to follow-up over 10 years. The VA outcomes of the excluded patients at each year of exit show the well reported pattern of initial VA gains followed by a decline after two years [20,21]. However, selection bias is inevitable in real-world outcome studies. Not all data were available but LOCF was not done to avoid skewed results as this is a long-term study. Moreover, the VA measurements were done in the clinical scenario using ETDRS letters and could be considered as a limitation. FA are not done routinely at regular intervals after initiation of treatment and retreatment is based on OCT and VA only. As these patients are the first group of patients initiated on anti-VEGF therapy, Moorfields Eye Hospital was still developing the service and fast-track services were in its infancy. Therefore the presenting vision may be lower than that of the patients presenting to clinic with neovascular AMD today.

In conclusion, the current study strongly suggests that by 10 years, in patients with neovascular AMD, good VA outcomes can be achieved and maintained with anti-VEGF therapy in clinical practice. The VA outcomes are influenced by baseline VA and final MA as the natural course of the disease continues to progress.

\section{Summary}

\section{What was known before}

- Anti-VEGF injections in clinical trial setting offer patients with nAMD previously unachievable improvement in vision. 
- Eyes with exudative AMD continue to remain at risk even in the late stages of therapeutic course.

\section{What this study adds}

- Current study strongly suggests that by 10 years, in patients with neovascular AMD, good VA outcomes can be achieved and maintained with anti-VEGF therapy in clinical practice.

- The VA outcomes are influenced by baseline VA and final MA as the natural course of the disease continues to progress.

Acknowledgements We would like to thank the Moorfields Medical Retina Group for their contribution and collation of patients. The research was supported by the NIHR Biomedical Research Centre at Moorfields Eye Hospital NHS Foundation Trust and UCL Institute of Ophthalmology.

\section{Compliance with ethical standards}

Conflict of interest SS reported receiving research grants from Novartis, Bayer, Allergan, Roche, Boehringer Ingelheim, and Optos Plc, travel grants from Novartis and Bayer, speaker fees from Novartis, Bayer, and Optos Plc, and attending advisory board meetings for Novartis, Bayer, Allergan, Roche, Boehringer Ingelheim, Optos Plc, and Heidelberg Engineering. PH reported receiving research grants from Novartis Allergan and Bayer, travel grants from Novartis Allergan and Bayer, speaker fees from Novartis Allergan and Bayer, and attending advisory board meetings for Novartis, Bayer, and Allergan. LN reported receiving speaker fees from Allergan. RH reports personal fees from Bayer, Novartis, Allergan and Ellex. PAK has received speaker fees from Heidelberg Engineering, Topcon, Carl Zeiss, Meditec, Haag-Streit, Allergan, Novartis and Bayer. He has served on advisory boards for Novartis and Bayer and has been external consultant for DeepMind and Optos. He is supported by a UK National Institute for Health Research (NIHR) Clinician Scientist Award (NIHR-CS-2014-12-23). SC, CA, $\mathrm{DM}, \mathrm{HK}$, and $\mathrm{BP}$ have no disclosures.

Publisher's note Springer Nature remains neutral with regard to jurisdictional claims in published maps and institutional affiliations.

\section{References}

1. Rosenfeld PJ, Brown DM, Heier JS, Boyer DS, Kaiser PK, Chung CY, Kim RY; MARINA Study Group. Ranibizumab for neovascular age-related macular degeneration. $\mathrm{N}$ Engl $\mathrm{J}$ Med. 2006;355:1419-31.

2. Michels S, Rosenfeld PJ, Puliafito CA, Marcus EN, Venkatraman AS. Systemic bevacizumab (Avastin) therapy for neovascular age-related macular degeneration twelve-week results of an uncontrolled open-label clinical study. Ophthalmology. 2005;112:1035-47.

3. Brown DM, Michels M, Kaiser PK, Heier JS, Sy JP, Ianchulev T, ANCHOR Study Group. Ranibizumab versus verteporfin photodynamic therapy for neovascular age-related macular degeneration: two-year results of the ANCHOR study. Ophthalmology. 2009;116:57-65.e5.

4. Heier JS, Brown DM, Chong V, Korobelnik JF, Kaiser PK, Nguyen $\mathrm{QD}$, et al. Intravitreal aflibercept (VEGF trap-eye) in wet age-related macular degeneration. Ophthalmology. 2012;119:2537-48.

5. Comparison of Age-related Macular Degeneration Treatments Trials (CATT) Research Group, Maguire MG, Martin DF, Ying GS, Jaffe GJ, Daniel E, Grunwald JE, et al. Five-year outcomes with anti-vascular endothelial growth factor treatment of neovascular age-related macular degeneration: the comparison of age-related macular degeneration treatments trials. Ophthalmology. 2016;123:1751-61.

6. Rofagha S, Bhisitkul RB, Boyer DS, Sadda SR, Zhang K, SEVEN-UP Study Group. Seven-year outcomes in ranibizumabtreated patients in ANCHOR, MARINA, and HORIZON: a multicenter cohort study (SEVEN-UP). Ophthalmology. 2013; 120:2292-9.

7. Holz FG, Tadayoni R, Beatty S, Berger A, Cereda MG, Cortez R, et al. Multi-country real-life experience of anti-vascular endothelial growth factor therapy for wet age-related macular degeneration. Br J Ophthalmol. 2015;99:220-6.

8. Cohen SY, Mimoun G, Oubraham H, Zourdani A, Malbrel C, Queré S, et al. Changes in visual acuity in patients with wet agerelated macular degeneration treated with intravitreal ranibizumab in daily clinical practice: the LUMIERE study. Retina. 2013;33:474-81.

9. Sunness JS, Bressler NM, Tian Y, Alexander J, Applegate CA. Measuring geographic atrophy in advanced age-related macular degeneration. Investig Ophthalmol Vis Sci. 1999;40:1761-9.

10. Wada I, Oshima Y, Shiose S, Kano K, Nakao S, Kaizu Y, et al. Five-year treatment outcomes following intravitreal ranibizumab injections for neovascular age-related macular degeneration in Japanese patients. Graefes Arch Clin Exp Ophthalmol. 2019;257: 1411-8.

11. Keenan TD, Vitale S, Agrón E, Domalpally A, Antoszyk AN, Elman MJ, et al. Visual acuity outcomes after anti-vascular endothelial growth factor treatment for neovascular age-related macular degeneration: age-related eye disease Study 2 Report Number 19. Ophthalmol Retina. 2019;4:3-12.

12. Fasler K, Moraes G, Wagner S, Kortuem KU, Chopra R, Faes L, et al. One- and two-year visual outcomes from the Moorfields agerelated macular degeneration database: a retrospective cohort study and an open science resource. BMJ Open. 2019;9:e027441.

13. Holz FG, Tadayoni R, Beatty S, Berger A, Cereda MG, Hykin P, et al. Key drivers of visual acuity gains in neovascular age-related macular degeneration in real life: findings from the AURA study. Br J Ophthalmol. 2016;100:1623-8.

14. Qin VL, Young J, Silva FQ, Conti FF, Singh RP. Outcomes of patients with exudative age-related macular degeneration treated with antivascular endothelial growth factor therapy for three or more years: a review of current outcomes. Retina. 2018;38: $1500-8$.

15. Kaiser PK, Singer M, Tolentino M, Vitti R, Erickson K, Saroj N, et al. Long-term safety and visual outcome of intravitreal aflibercept in neovascular age-related macular degeneration: VIEW 1 extension study. Ophthalmol Retina. 2017;1:304-13.

16. Chakravarthy U, Harding SP, Rogers CA, Downes SM, Lotery AJ, Culliford LA, et al. Alternative treatments to inhibit VEGF in age-related choroidal neovascularisation: 2-year findings of the IVAN randomised controlled trial. Lancet. 2013;382:1258-67.

17. Grunwald JE, Daniel E, Huang J, Ying GS, Maguire MG, Toth $\mathrm{CA}$, et al. Risk of geographic atrophy in the comparison of agerelated macular degeneration treatments trials. Ophthalmology. 2014;121:150-61. 
18. Sarraf D, London NJ, Khurana RN, Dugel PU, Gune S, Hill L, et al. Ranibizumab treatment for pigment epithelial detachment secondary to neovascular age-related macular degeneration: post hoc analysis of the HARBOR study. Ophthalmology. 2016;123: 2213-24.

19. Kaynak S, Kaya M, Kaya D. Is there a relationship between use of anti-vascular endothelial growth factor agents and atrophic changes in age-related macular degeneration patients? Turk J Ophthalmol. 2018;48:81-4.
20. Lee AY, Lee CS, Butt T, Xing W, Johnston RL, Chakravarthy U, et al. UK AMD EMR USERS GROUP REPORT V: benefits of initiating ranibizumab therapy for neovascular AMD in eyes with vision better than 6/12. Br J Ophthalmol. 2015;99: 1045-50.

21. Talks JS, Lotery AJ, Ghanchi F, Sivaprasad S, Johnston RL, Patel $\mathrm{N}$, et al. First-year visual acuity outcomes of providing aflibercept according to the view study protocol for age-related macular degeneration. Ophthalmology. 2016;123:337-43. 